\title{
Controversias sobre ADHD y metilfenidato en discusiones sobre medicalización en Argentina y Brasil
}

I 1 Eugenia Bianchi, 2 Silvia Faraone, ${ }^{3}$ Francisco J. Ortega,

${ }^{4}$ Valéria Portugal Gonçalves, ${ }^{5}$ Rafaela Teixeira Zorzanelli I

Resumen: El trabajo tiene como objetivo ofrecer un análisis sistemático de algunos resultados de investigaciones realizadas en Argentina y Brasil relacionadas con el uso del metilfenidato, su prescripción y la regulación de la venta. También examinamos las discusiones entre investigadores y profesionales que asisten a personas diagnosticadas con TDAH en ambos países. El artículo integra dos campos de investigación. En Argentina se analizaron artículos de prensa, datos numéricos de organizaciones oficiales y profesionales y entrevistas semiestructuradas de profesionales de salud individuales y grupales realizadas entre 2007 y 2011. En Brasil se analizaron las revistas científicas, la observación participante y los profesores de las escuelas y los profesionales de la salud. Las conclusiones incluyen que los movimientos sociales en ambos países se han organizado y articulado, en un intento de promover el debate sobre la medicalización de la infancia y su despliegue en la sociedad.

> Palabras-clave: TDHA; metilfenidato; Argentina; Brasil; medicalización.
1 Instituto de Investigaciones Gino Germani, Facultad de Ciencias Sociales, Universidad de Buenos Aires. Buenos Aires, Argentina (eugenia.bianchi@ yahoo.com.ar)

${ }^{2}$ Instituto de Investigaciones Gino Germani, Facultad de Ciencias Sociales, Universidad de Buenos Aires. Buenos Aires, Argentina (silfaraone@gmail.com).

${ }^{3}$ Instituto de Medicina Social, Universidade do Estado do Rio de Janeiro. Rio de Janeiro-RJ, Brasil (fjortega2@gmail.com).

${ }^{4}$ Instituto de Medicina Social, Universidade do Estado do Rio de Janeiro. Rio de Janeiro-RJ, Brasil (goncalves.vp@gmail.com).

${ }^{5}$ Instituto de Medicina Social, Universidade do Estado do Rio de Janeiro. Rio de Janeiro-RJ, Brasil (rtzorzanelli@gmail.com).

Recibido en: 06/07/2016 Aprovado en: 01/12/2016 


\section{Introducción}

En este artículo presentamos resultados de investigaciones en Argentina (FARAONE; BIANCHI, 2016) y Brasil (ORTEGA; ZORZANELLI; GONÇALVES, 2016), realizadas en los últimos ocho años, en las cuales profundizamos el análisis del diagnóstico del Trastorno por Déficit de Atención e Hiperactividad (TDAH) y exponemos las disputas que acarrea a partir de la conformación y el devenir de organizaciones profesionales en ambos países, en un intento por promover el debate sobre la medicalización de la infancia y su despliegue en la sociedad. Contextualizamos la problemática con información vinculada al metilfenidato, principal fármaco prescripto para este diagnóstico (SINGH, 2008), la regulación de su prescripción y venta, y la discusión que se presenta entre diferentes investigadores y profesionales que asisten a los niños y adolescentes que se diagnostican con TDAH en ambos países.

Si bien los objetivos de las investigaciones realizadas por los equipos de Argentina y Brasil no fueron formulados para una comparación ulterior, surgió a partir de varios encuentros, particularmente a través del Programa de Centros Asociados (Ministerio de Educación, Argentina -CAPES- Ministério da Educação, Brasil), que los trabajos de ambos grupos concluían en resultados interesantes a ser sistematizados conjuntamente a fin de exponer una problemática de relevancia creciente en Salud Pública, e identificada por los equipos como central para el debate en la agenda de los Estados y de la Región.

En los últimos años, el TDAH se ha tornado un diagnóstico en extraordinario ascenso en el escenario mundial (CONRAD; BERGEY, 2014) y se viene configurando como un importante tema de discusión en Salud Pública en diversos países. Tales discusiones abarcan la polémica en torno de la existencia del diagnóstico suscitada entre los profesionales clínicos e investigadores, y la creciente utilización de metilfenidato alrededor del mundo para el tratamiento del TDAH (SINGH, 2008).

En este contexto diversas investigaciones se pronunciaron sobre la cuestión del trastorno y prescripción del metilfenidato, produciendo significativas contribuciones para el debate. Esta problemática es recibida con preocupación por organizaciones profesionales que discuten la medicalización de la vida, cuestionando el diagnóstico y la prescripción de psicofármacos para síntomas que consideran comportamientos esperados en la infancia y adolescencia. Los estudios 
científicos exponen resultados de prevalencia de TDAH de amplia variabilidad y

altas tasas de comorbilidad -más allá de las diferentes percepciones culturales- lo que para algunos críticos no parece aportar consistencia respecto a la exactitud de criterios diagnósticos (TIMIMI et al., 2004; TIMIMI; RADCLIFFE, 2005; MONCRIEFF; TIMIMI, 2010; WEDGE, 2015).

A la vez, importantes centros de investigación sostienen que a pesar del incremento del número de niños y adolescentes diagnosticados y del uso de metilfenidato, el TDAH estaría siendo subdiagnosticado y subtratado, por insuficiente información en los profesionales, siendo la población pobre la más perjudicada por este desconocimiento (MATTOS; ROHDE; POLANCZYK, 2012).

Por último, si bien Conrad y Bergey (2014) situaron las resistencias a la medicalización del TDAH como un elemento relevante para analizar la globalización del cuadro, no existen trabajos que estudien los agrupamientos de estos movimientos de profesionales de diferentes países. Este trabajo aporta a esta área de vacancia con resultados de dos países cuya relevancia en el Cono Sur resulta insoslayable.

\section{Metodología}

En este artículo sistematizamos y analizamos información de investigaciones realizadas en Argentina y Brasil por dos equipos pertenecientes al Instituto de Investigaciones Gino Germani de la Universidad de Buenos Aires y al Instituto de Medicina Social de la Universidad Estadual de Río de Janeiro. Ambos equipos trabajaron con una metodología cualitativa basada en entrevistas en profundidad a informantes clave, empleando información primaria y secundaria comprendida entre 2007-2014.

Los resultados de Argentina provienen de fuentes que incluyen bibliografía especializada nacional e internacional, artículos periodísticos sobre la temática (de diarios de mayor tirada), datos numéricos oficiales y de organizaciones profesionales (ARIZAGA; FARAONE, 2008), y 65 entrevistas semiestructuradas individuales y grupales a profesionales de la salud, realizadas en dos etapas: 35 de octubre a diciembre de 2007 (ARIZAGA; FARAONE, 2008), y de abril a junio 2008; y 30 entre agosto de 2009 y marzo de 2011 (BIANCHI, 2014). 
Para la muestra de entrevistas se siguió un criterio intencional no probabilístico, orientado a profesionales de la salud con inserción clínica, docente y de investigación del subsector público, la seguridad social y del subsector privado. Las entrevistas se realizaron en cuatro jurisdicciones: Tierra del Fuego, Corrientes, Salta y Ciudad de Buenos Aires. Se efectuaron además entrevistas a informantes clave del campo de la salud y la educación y a agentes de propaganda médica de la industria farmacéutica.

Para el caso de Brasil, los resultados corresponden a investigaciones que relevaron artículos periodísticos y revistas sobre la temática. Se realizó un análisis bibliográfico acerca del TDAH y el metilfenidato en las bases Scielo y PubMed (ORTEGA; ZORZANELLI; GONÇALVES, 2016), y en periódicos y revistas orientadas al público en general. También se analizaron 103 publicaciones brasileñas en el período 1998-2008, incluyendo 72 reportajes en periódicos y revistas de gran circulación y 31 artículos de periódicos de psiquiatría sobre los usos de la Ritalina ${ }^{\circledR}$ (ITABORAHY; ORTEGA, 2010). Asimismo se incluyó un relevamiento a través de seis entrevistas semiestructuradas y observación participante en nueve reuniones semanales de profesores y tres reuniones mensuales entre la coordinadora escolar y los profesionales de salud de la red pública próxima a una escuela de la municipalidad de Niterói, del Estado de Rio de Janeiro durante el segundo semestre de 2011 (CHAGAS, 2013).

Para el procesamiento de datos primarios y secundarios, ambos equipos, emplearon métodos analítico-interpretativos y de triangulación de fuentes y resultados. Asimismo, ambos equipos a través de sus trabajos de investigación se atuvieron a normativas y resguardos éticos nacionales e internacionales vigentes para los estudios sobre salud humana (ARGENTINA, 2006; 2011; BRASIL, 2012). Se utilizaron consentimientos informados para la realización de las entrevistas. Los mismos se documentaron a través de formularios escritos y fechados que se entregaron a los involucrados, explicitando que la información obtenida en la entrevista revestiría carácter absolutamente confidencial y anónimo. Los planes de trabajo de las investigaciones y consentimientos informados fueron evaluados y avalados por los Comités de Ética de los respectivos Institutos de Investigaciones.

Por último, informamos no tener conflictos de intereses políticos y/o financieros asociados a fuentes de financiamiento, patentes o propiedad, provisión de materiales y/o bienes y equipamientos. Exponemos entonces, en 
este artículo, una sistematización a partir de datos cualitativos y cuantitativos en la que convergen diferentes fuentes puestas en diálogo y articulación.

\section{Las controversias sobre el metilfenidato y el incremento en la venta}

Las controversias que surgen respecto de la prescripción de psicofármacos en niños tienen un componente singular, que se suscita porque las decisiones terapéuticas que los atañen no son tomadas por ellos mismos, y están, en cambio, influidas por los adultos, sean sus padres, docentes o terapeutas. A pesar que el uso de estimulantes en niños preexiste a la tipificación del TDAH (MAYES; BAGWELL; ERKULWATER 2008), la prescripción del metilfenidato para este diagnóstico supone una zona propia de críticas, por las reacciones adversas documentadas (BIGNONE; SERRATE; DIEZ, 2007), y por cuestiones referentes al uso para el mejoramiento del desempeño escolar y el posible encubrimiento de aspectos pedagógicos, familiares y sociales que traería aparejado (BARROS, 2014).

El metilfenidato es considerado hoy la droga de primera elección entre las perspectivas que abogan por el tratamiento medicamentoso, siendo el psicoestimulante más frecuentemente prescripto para el tratamiento del TDAH (SINGH, 2008; BIGNONE; SERRATE; DIEZ, 2007; LANGE et al., 2010; BARKLEY, 2006). Conrad y Bergey ubican a los fármacos empleados en la terapéutica del TDAH como pieza clave en la expansión potencial del mercado farmacéutico a nivel global (CONRAD; BERGEY, 1014).

Bajo el nombre comercial de Ritalina ${ }^{\circledR}$ y Concerta $^{\circledR}$, el metilfenidato es el estimulante de mayor venta en Brasil para el tratamiento del TDAH. En este país, dos laboratorios comercializan la sustancia, bajo la forma de comprimidos de liberación rápida, lenta y cápsulas de liberación modificada completando, en total, ocho presentaciones y siete concentraciones diferentes. En Argentina el metilfenidato también es la droga de primera elección y mayor venta. Específicamente, la comercializan cuatro laboratorios, en 16 presentaciones y siete concentraciones diferentes, bajo formas de liberación controlada prolongada, y modificada.

Aunque la comercialización de este psicofármaco se remonta a la década del 1950 (HEALY, 2002), está documentado que la asociación cada vez más directa entre metilfenidato y TDAH se relaciona con el aumento internacional en la producción y el consumo del psicoestimulante a partir de la década de 1990. 
De acuerdo a datos de la Organización de las Naciones Unidas, la producción mundial de metilfenidato aumentó $1200 \%$ entre 1990 y 2006 (INCB, 2008). Según la Junta Internacional de Control de Estupefacientes (Internacional Narcotics Control Board, INCB) el consumo global pasó de 21,8 toneladas en 2002 a 35,8 toneladas en 2006 (INCB, 2008). Entre 2005 y 2009 el consumo mundial sostuvo su escalada, alcanzando las 40 toneladas, lo que significa un incremento del 30\% (INCB, 2012).

Para el caso de Brasil, desde la década del '90 se verificó un aumento del consumo, alcanzando un volumen de $23 \mathrm{~kg}$. para el año 2000. Por otra parte, la producción brasileña anual pasó de $40 \mathrm{~kg}$. en 2002, a $226 \mathrm{~kg}$. en 2006, acrecentado por una importación de $91 \mathrm{~kg}$. (INCB, 2008). Ya para el 2011, la producción nacional presentó un valor menor, con $81 \mathrm{~kg}$. aunque se importaron $210 \mathrm{~kg}$. adicionales (INCB, 2013). No existen datos más recientes dado que Brasil, así como otros importantes países productores (Alemania, Austria, Canadá, China, Estados Unidos, Francia, Japón, Países Bajos y Paquistán) no reportaron a tiempo las estadísticas de las sustancias psicotrópicas a la INCB para la elaboración del informe de 2012 (INCB, 2013). Este fracaso en la remisión de resultados fue objeto de preocupación de la Junta en sus últimos informes anuales (INCB, 2012; 2013).

Considerando una franja de tiempo más amplia, Barros (2014) observó que la tasa de comercialización de metilfenidato en Brasil fue más significativa que la cifra mundial. Verificó que entre 1996 y 2012 la cantidad de metilfenidato producido en territorio nacional e importado, pasó de 9 kilos a 578 kilos, representando un aumento del $6322 \%$, en comparación a la fabricación mundial de metilfenidato entre 1998 y 2012, que varió de 13.493 kilos a 63.236 kilos, significando un aumento de 356,94\%.

Para el caso de Argentina no se publican estadísticas oficiales sobre el diagnóstico de TDAH, ni sobre los fármacos prescriptos ni consumidos. Sin embargo diferentes profesionales entrevistados, así como artículos periodísticos refieren un incremento sostenido en la prescripción y consumo del metilfenidato en el siglo XXI. Argentina no produce metilfenidato, aunque importa la monodroga y elabora el principio activo en distintos laboratorios. Se comercializa internamente y exporta a algunos países limítrofes. 
Desde diferentes fuentes secundarias que toman a la Administración Nacional de Medicamentos, Alimentos y Tecnología Médica (ANMAT) como origen de los datos (CARBAJAL, 2007; MANIOWICZ, 2011), y con información solicitada directamente al Departamento de Psicotrópicos y Estupefacientes de esta administración, se obtuvieron datos de importación anual para la década 20032013. La información recabada sindica a 2004 como el año en que la cantidad de monodroga importada al país inicia su escalada, pasando de 23,7 kilos en el período previo, a 40,4 kilos, en una tendencia creciente que se consolidó desde entonces, alcanzando los 55,23 kilos en 2010. En 2011 la importación ascendió a 85,99 kilos; y aunque en 2012 indicaban una baja a 38,15 kilos, para 2013 había vuelto a aumentar, esta vez hasta los 100,55 kilos. A pesar de la oscilación de los valores interanuales, a efectos de marcar una tendencia, los datos congruyen en un alza sostenida en la importación del metilfenidato. Se observó que al igual que en Brasil, esta tendencia es consistente con la global, como también lo marca la INCB en los informes de 1995 y 2006 (INCB, 1995; 2006).

Un aspecto documentado en Brasil y Argentina es la periodicidad en la dispensación del metilfenidato. En Brasil, según la Agência Nacional de Vigilância Sanitária (ANVISA), la comercialización se reduce hacia fines del año lectivo, lo que podría leerse como una disminución en la prescripción durante las vacaciones escolares (ANVISA, 2010). En Argentina el metilfenidato también describe una estacionalidad relacionada con el año lectivo. De acuerdo con datos de expendio suministrados al equipo de Argentina por la Confederación Farmacéutica Argentina (COFA), para el trienio 2005-2007 el volumen de dispensación se mantiene alto durante el ciclo de cursada, y cae significativamente en el período de vacaciones escolares (ARIZAGA; FARAONE, 2008).

Para Argentina, añadimos lo documentado en las entrevistas a profesionales, quienes refieren una planificación flexible de la prescripción del psicofármaco, orientada por las actividades concretas que realiza el niño durante el día. Estas actividades varían en función de las características del ciclo escolar (doble escolaridad, días de la semana o fin de semana, época lectiva o vacacional, cursada con otros niños o clases individuales con psicopedagoga, entre otros), y de la gravedad del cuadro. Así, enfatizan que la periodicidad y dosis en el suministro del mismo se adecuan a las variabilidades individuales (BIANCHI, 2014). 
Esta prescripción del psicofármaco, que varía y se modifica de acuerdo a las necesidades que el medio impone, está en línea con una terapéutica farmacológica que es considerada como un apoyo adicional, capaz de ampliar las probabilidades de transitar espacios y sostener relaciones familiares, sociales, afectivas, académicas y laborales a aquellos que, en virtud de sus fallas en la "administración del yo" (ROSE, 2006), llevan adelante una vida que debe ser administrada por otros.

\section{Metilfenidato y resistencias a la medicalización}

Profesionales de la educación y de la salud mental brasileños y argentinos observaron con reservas el posicionamiento ascendiente del metilfenidato en el mercado farmacológico en los últimos años, en virtud de diferentes registros que, ya sea que estén enfocados en la producción, la venta o el consumo, convergen en señalar una tendencia alcista en los últimos años. Este hecho, ligado al hallazgo de la fuerte presencia de la industria farmacéutica en las investigaciones sobre TDAH y metilfenidato en ambos países (BIANCHI et al., 2016), ha llevado a críticas acerca de los resultados de las investigaciones sobre el trastorno, la validez del diagnóstico y su tratamiento medicamentoso.

Con una extensa tradición psicoanalítica, y a pesar de la creciente influencia de otras corrientes y perspectivas diagnósticas y terapéuticas en el ámbito de la salud mental, el lugar que ocupa el psicoanálisis en Brasil y Argentina -y en las Ciudades de Buenos Aires y Rio de Janeiro como perfiles centrales y dominantes en cada país- los constituye en singularidades fenoménicas en relación con otros países europeos y latinoamericanos. En ambas ciudades el psicoanálisis mantiene numerosos adeptos, y sostiene modalidades específicas de articulación y divergencia con otras prácticas clínicas, tanto en el subsector público como en el privado. La diseminación en la sociedad se expresa además, en la cantidad y peso de sus instituciones, la profusión de conceptos psicoanalíticos en el lenguaje cotidiano, que lo constituyen en voz pública autorizada para opinar acerca de variados temas en los medios de comunicación.

Entre los profesionales brasileños, se destacan educadores, psicólogos y psicoanalistas como los especialistas que sostienen la necesidad de ampliar la mirada sobre quienes presentan dificultades de aprendizaje, inquietud y falta de atención. Argumentan que la indicación de metilfenidato debe reservarse a los 
casos más severos y, dado el deterioro tanto académico como laboral que deriva de los problemas de conducta, el abordaje debe ser multidisciplinario y psicosocial.

En este país, estas posturas críticas se organizaron en movimientos de profesionales, entre los cuales se destaca el Fórum sobre Medicalização da Educação e da Sociedade (www.medicalizacao.org.br), integrado por diversos investigadores y profesionales, siendo los consejos estaduales de psicología de Rio de Janeiro y de São Paulo importantes articuladores del movimiento. El Fórum fue fundado por una médica pediatra e investigadora del Departamento de Pediatría de la Universidade Estadual de Campinas (UNICAMP) en el estado de São Paulo. Entre los logros del Fórum se cuenta la participación en la creación del decreto de la Secretaría Municipal de Salud de São Paulo, para regular la prescripción de metilfenidato en casos de TDAH identificados en la ciudad y que se inspiró en el protocolo de abordaje de agitaciones y dispersiones en niños y adolescentes en la Ciudad de Campinas (SÃO PAULO, 2014). El Fórum surgió en 2010, a partir de un espacio de debate construido en São Paulo para la discusión de programas para la dislexia y más tarde para el TDAH. La tónica de esos programas era considerada un retroceso para afrontar las dificultades de la escolarización, dado que promovía una reducción de los problemas educacionales a una supuesta patología del sujeto. Desde su creación, el Fórum establece vínculos con diversos núcleos y entidades en el país y el exterior (FÓRUM..., 2013).

En Argentina, las polémicas en torno a la importación y consumo de metilfenidato, el incremento de casos de TDAH en niños, y el empleo preponderante del manual DSM dieron pie simultáneamente a una serie de reuniones científicas y profesionales, en forma de congresos, jornadas y simposios. Desde posturas muy diferentes entre sí, se publicaron compilaciones de artículos de profesionales (JOSELEVICH, 2003; MOYANO WALKER, 2004; JANIN, 2004; STIGLITZ, 2006).

En este país, los profesionales que critican la terapéutica medicamentosa como opción semiautomática también provienen de diferentes disciplinas y especialidades: neurólogos y psiquiatras infantiles, psicopedagogas, pediatras, psicólogos y psicoanalistas. Estos profesionales de la salud, la educación y las ciencias sociales llevan realizados cuatro simposios bianuales en la Ciudad de Buenos Aires desde 2007, e impulsan otras reuniones profesionales en diferentes ciudades del país, promoviendo la formación de agrupaciones locales con características semejantes a las del Fórum (UNTOIGLICH, 2014). 
El Comité Ejecutivo del Forum Infancias consta de nueve miembros, está integrado en su totalidad por especialistas en psicoanálisis. Sus argumentaciones hacen foco en los efectos adversos que provoca la administración de metilfenidato en niños, e integran esos efectos nocivos en una mirada más amplia, en la cual el etiquetamiento en términos de enfermedad, déficit y trastorno se continúa con el recurso al fármaco (www.foruminfancias.com.ar/comision-directiva/). Este Forum, junto a otras organizaciones sociales y profesionales vinculadas a la sanción de la Ley de Salud Mental (Ley 26.657/2010), son actores relevantes en las disputas presentadas por ciertos sectores del Honorable Congreso de la Nación, que avanzan en distintas instancias de promulgación de leyes por patologías, entre las cuales existe un proyecto en relación al TDAH.

Las asociaciones de profesionales de ambos países han forjaron vínculos entre sí. En mayo de 2012, en la Feria del Libro de Buenos Aires, convocaron a profesionales de la salud y la educación en el marco de la Campaña Internacional Stop DSM! De esta convocatoria participaron representantes del Forum Infancias y Fórum sobre Medicalização da Educação e da Sociedade. En la misma desarrollaron las posiciones de ambos foros, los temas que trabajan conjuntamente, y propusieron el armado de redes para pensar el sufrimiento infantil, y ofrecer la escucha a niños, adolescentes y adultos a su cargo. En el encuentro, y en el marco del panel La infancia medicalizada. Situación en Argentina y Brasil, se relanzó la Carta de la UNASUR, redactada en conjunto a mediados del año anterior.

Inscripta en lo que denomina un movimiento de carácter ético y político, guiado por el rigor científico y la defensa intransigente de la vida, la Carta de la UNASUR concluye que los integrantes de ambos forum no acuerdan con el uso del DSM-IV y del 5 (en elaboración ese momento) en ninguna persona, pero especialmente en niños y adolescentes; que a todo niño y adolescente se le debe garantizar acceso a atención médica, psicológica, fonoaudiológica y pedagógica sin necesidad de certificado o diagnóstico, por su sola condición ciudadana; y que la educación y salud públicas de calidad son derechos de todos y deber del Estado.

Además del vínculo mutuo, estas asociaciones articulan su labor con el grupo español Espai Freud (impulsores de la campaña internacional Stop DSM!), el Forum por uma abordagem não medicalizante nem patologizante da educação de Portugal, la agrupación francesa Pas de zéro de conduite y otras organizaciones en México y Chile (UNTOIGLICH, 2014). 


\section{Reglamentaciones, consensos y disensos}

En Argentina, el Forumadd publicó en 2005 el Consenso de expertos del área de la salud, dirigido al Ministerio de Salud de la Nación, y firmado entonces por alrededor de 200 renombrados profesionales de distintas especialidades. El documento critica la extensión del diagnóstico y la prescripción de metilfenidato, resaltando algunos efectos adversos. Propone que los niños sean evaluados por expertos, y que la medicación sea un último recurso, valorando el contexto familiar y social del niño en la evaluación, y entendiendo que la información sobre el TDAH en los medios masivos de comunicación debe ser restringida, debido a las controversias entre los profesionales acerca de la existencia del cuadro (www.foruminfancias.com.ar/consenso/). Fue elevado además un pedido de informes al Poder Ejecutivo Nacional, tratado al año siguiente en la Honorable Cámara Nacional de Diputados (ARGENTINA, 2006).

Este Consenso ameritó la réplica de otras posturas, expresadas en la publicación en el sitio www.tdah.org.ar de la traducción del original en inglés del Consenso Internacional sobre ADHD, de 2002, que considera al TDAH como un trastorno válido, y sostiene que el pedido de restricción de divulgación de información sobre este es una limitación a la libertad de expresión.

Según Conrad y Bergey (2014), los consensos publicados la década pasada, destinados a promover el diagnóstico y brindar fundamentos a los profesionales, fueron creados desde Estados Unidos para una audiencia global. Si bien reconocían las controversias que ya se multiplicaban en torno al TDAH, también planteaban elementos para concluir los debates, ya que invocaban evidencia científica para el reconocimiento del TDAH como un trastorno real. En particular, el mencionado consenso de 2002, autodefinido como Internacional, contaba apenas con un 12\% de firmantes no provenientes de Norteamérica. Otro aspecto de los consensos internacionales es la fundamentación del diagnóstico en los criterios del DSM.

De todas maneras, ya para 2007 en Argentina una disposición de ANMAT ordenaba a los laboratorios que comercializan metilfenidato que incluyeran advertencias, precauciones y contraindicaciones en los prospectos (ANMAT, 2007).

En Argentina las críticas al manual DSM están asociadas a la Ley de Salud Mental 26.657 (ARGENTINA, 2010). Esta ley, vigorosamente resistida por algunas asociaciones psiquiátricas, es de avanzada en relación a estándares internacionales, teniendo como objetivo asegurar "el pleno goce de los derechos 
humanos de aquellas [personas] con padecimiento mental" (artículo 1). Esta definición generó profusos debates legislativos, porque emplea un concepto que desplaza por un lado, la noción de trastorno mental, y por otro la hegemonía médico-farmacéutica (FARAONE, 2013). La ley aboga por el abordaje interdisciplinario (artículo 8), el uso de fármacos con fines exclusivamente terapéuticos, y no como castigo (artículo 12). En relación al TDAH, la ley fortaleció a los grupos que cuestionan la patologización de la infancia.

En Brasil, como efecto de las discusiones suscitadas, la Ordenanza 986/2014 emitida por la Secretaría Municipal de Salud de São Paulo encendió el debate actualizando las cuestiones más polémicas y polarizadas entre diferentes posturas. São Paulo es la capital del estado de la región sudeste del país y la ciudad más populosa de Brasil, alojando el parque industrial más importante del país. Según la Ordenanza, los usuarios sospechados de tener un diagnóstico de TDAH deben derivarse a Centros de Atenção Psicossocial Infantil (CAPSi), y en localidades donde no existan tales servicios, los usuarios deben enviarse a unidades de tratamiento combinado, con intervenciones terapéuticas de naturaleza psicosocial y educativa de la red pública (FÓRUM..., 2013). Esta ordenanza procura reglamentar la prescripción de metilfenidato a niños y adolescentes con TDAH.

Los CAPSis integran la Rede de Atenção Psicossocial junto con dispositivos de atención y tratamiento para pacientes con trastorno mental bajo la modalidad de internación, con camas en hospitales generales, residencias terapéuticas y atención básica, cuyo circuito fue diseñado en conformidad con la política de desinstitucionalización de la atención psiquiátrica en el país (Brasil 2011). Aunque la política de salud mental de orientación basagliana rige desde 2001 a través de la Ley Nacional 10.216/01 (CSILLAG, 2001; BRASIL, 2005), la misma es objeto de fuerte resistencia, coincidentemente con el caso argentino, de la Associação Brasileira de Psiquiatria, frecuentemente posicionada contra sus determinaciones. La asociación alega que la disminución de internaciones psiquiátricas preconizada por la "ideología" de la desinstitucionalización desatiende a la población.

Como reacción a la Ordenanza de São Paulo, la Associação Brasileira de Psiquiatria (ABP) publicó la Carta à População, declarándose contraria a su implementación (www.abp.org.br). El documento fue firmado por 10 sociedades y asociaciones médicas regionales de medicina, psiquiatría y neurología, además de la Sociedade Brasileira de Neuropsicologia, la Sociedade Brasileira de 
firmaron más de 50 profesionales médicos, psiquiatras y psicólogos, magísteres y doctores, profesores universitarios y presidentes de asociaciones profesionales.

En el documento, la ABP alega que la Ordenanza burocratiza el acceso digno al tratamiento, principalmente de la población socialmente menos favorecida, perjudicando la sistematización científica "de manera mistificadora e indigna" (ABP, 2014). Argumenta además que "es una obstrucción abusiva del acceso al tratamiento farmacológico por parte de la población de menores ingresos, e impone restricciones al pleno ejercicio y autonomía de la medicina y de la ciencia brasileña” (ABP, 2014).

Por otro lado, la iniciativa de São Paulo recibió el apoyo de la Associação Brasileira de Saúde Mental (www. abrasme.org.br) considerándola una medida ejemplar, en total acuerdo con una clínica de comprensión ampliada sobre el problema. La ABRASME sigue los preceptos de la psiquiatría democrática italiana de Basaglia, y apoya a trabajadores de salud mental, y el desarrollo de la formación, enseñanza e investigación en salud mental. La asociación también tiene como objetivo promover la coordinación entre los centros de formación, la enseñanza y los servicios de investigación y salud mental, para fortalecer y ampliar el diálogo con las comunidades científicas y técnicas y con los servicios de salud, el gobierno y las organizaciones no gubernamentales, y la sociedad civil (ABRASME, 2010; 2014).

\section{Conclusiones}

Respecto del metilfenidato, en ambos países se documentó el incremento en la venta del psicoestimulante en años recientes, y una periodicidad análoga en las ventas interanuales, coincidiendo el alza con los ciclos lectivos. También documentamos que las críticas a la venta de metilfenidato, en el marco de una configuración diagnóstica fuertemente cuestionada como el TDAH, fueron pilares para la conformación de organizaciones de profesionales que en la actualidad sostienen esas posturas.

Tanto Buenos Aires, como São Paulo y Rio de Janeiro aglutinan renombrados referentes en docencia, investigación y práctica clínica en salud mental, con posicionamientos críticos respecto del diagnóstico de TDAH y la prescripción de metilfenidato. En estas ciudades el psicoanálisis excede la órbita de la clínica 
en salud mental, imbricándose en una cultura urbana más amplia, siendo esa difusión más pronunciada en el caso de Argentina. En este contexto una diferencia observada entre ambas organizaciones de profesionales se da en la formación de las cúpulas, que en Argentina tienen una especialización psicoanalítica excluyente, mientras que en Brasil están también asociadas a otros perfiles, tales como la pediatría, pedagogía y salud colectiva.

Por otra parte, destacamos que lo analizado en Argentina y Brasil para el TDAH complejiza las consideraciones señaladas por Conrad y Bergey acerca de la regulación legal sobre prescripción y venta de psicofármacos. Los autores establecen que los países que imponen restricciones legales para el uso de estimulantes suponen obstáculos para la penetración de las compañías farmacéuticas e, inversamente, los países que alivianan esas condiciones legales resultan más accesibles a estas empresas (CONRAD; BERGEY 2014). En Brasil y Argentina las normativas nacionales existentes, aunque contemplan prohibiciones, restricciones y una perspectiva de derechos para las personas que son diagnosticadas con alguna condición de salud mental, no han redundado hasta el momento, en una limitación o desaliento sostenidos a la venta creciente del metilfenidato.

Los resultados aquí expuestos documentan procesos que avanzan en el accionar coordinado entre organizaciones de profesionales de Argentina y Brasil, y su confluencia en relación a la crítica al diagnóstico de TDAH y a la prescripción de metilfenidato, perfilándose encuentros y actividades comunes conducentes a la profundización de una lectura regional respecto de estos procesos. El devenir de estos encuentros constituye un escenario abierto y en formación, por lo que lo documentado hasta aquí para Argentina y Brasil, y su inscripción analítica en procesos, contextos y perspectivas más amplias provee elementos que aportan a la reflexión crítica acerca de problemas y fenómenos que, como el del incremento del consumo del metilfenidato, y su asociación al diagnóstico del TDAH, se posicionan con relevancia creciente en la agenda de salud pública regional. ${ }^{1}$

\section{Referencias}

ADMINISTRACIÓN NACIONAL DE MEDICAMENTOS, ALIMENTOS Y TECNOLOGÍA MÉDICA (ANMAT). Disposición 2123/2007. Trastornos por Déficit de Atención con Hiperactividad: Se modifican prospectos de especialidades medicinales para tratar esta patología. Boletín para profesionales, v. 15, n. 1, p. 3-7, 2007. Disponible 
en: <http://www.anmat.gov.ar/Publicaciones/Boletines/Profesionales/Boleprof_Febrero _2007.pdf>. Aceso: 28 dez 2015.

AGÊNCIA NACIONAL DE VIGILÂNCIA SANITÁRIA (ANVISA). Relatório 2009 - Sistema Nacional de Gerenciamento de Produtos Controlados (SNGPC). Rio de Janeiro: ANVISA, 2010.

ARGENTINA. Cámara Nacional de Diputados, Sesiones Ordinarias. Orden del día n 750 , 2006. Comisión de Acción Social y Salud Pública, de Educación y de Familia, Mujer, Niñez y Adolescencia. Disponible en: <http://www4.hcdn.gov.ar/dependencias/dcomisiones/ periodo-124/124-750.pdf>. Acceso: $28 \mathrm{dez} 2015$.

Ley Nacional de Salud Mental n²6.657 [2 dic 2010]. Honorable Congreso de la Nación Argentina. Disponible en: <http://www.infoleg.gob.ar/infolegInternet/ anexos/175000-179999/175977/norma.htm>.

- Resolución $D n^{\circ}$ 2857, de 11 de diciembre de 2006. Lineamientos para el comportamiento ético en las Ciencias Sociales y Humanidades. Comité de Ética - Consejo Nacional de Investigaciones Científicas y Técnicas (CONICET). Ministerio de Educación, Ciencia y Tecnología. Disponible en: <http://www.conicet.gov.ar/wp-content/uploads/ RD-20061211-2857.pdf>.

- Resolución $n^{\circ}$ 1480, de 13 de septiembre de 2011. Guía de Buenas Prácticas Clínicas de Investigación en Salud Humana. Ministerio de Salud. Disponible en: <http:// www.infoleg.gov.ar/infolegInternet/anexos/130000-134999/134488/norma.htm>

ARIZAGA, M.C., FARAONE, S. La medicalización de la infancia. Niños, escuela y psicotrópicos. Buenos Aires: UBA-SEDRONAR, 2008. Disponible en: <http://scripts. minplan.gob.ar/octopus/archivos.php?file =4270>. Acceso: 4 may. 2016.

ASSOCIAÇÃO BRASILEIRA DE PSIQUIATRIA (ABP). Carta Aberta à População. Disponible en: http://www.abp.org.br/portal/carta-aberta-a-populacao/>. Acceso: 5 ago 2014. ASSOCIAÇÃO BRASILEIRA DE SAÚDE MENTAL (ABRASME). Estatuto da Associação Brasileira de Saúde Mental. Assembleia Geral da ABRASME, 2010. Disponible en: http:// www.abrasme.org.br/conteudo/view?ID_CONTEUDO=641. Acceso: 28 dic 2015.

Transtorno de déficit de atenção e hiperatividade (ADHD) e a iniciativa exemplar da SMS de São Paulo. 2014. Disponible en: <https://abrasmesp.wordpress.com/2014/07/23/ nota-abrasme-transtorno-de-deficit-de-atencao-e-hiperatividade-tdah-e-a-iniciativaexemplar-da-sms-de-sao-paulo/>. Acceso: 28 dic 2015.

BARKLEY, R. Attention-Deficit Hyperactivity Disorder. A handbook for diagnosis and treatment. $3^{\text {rd }}$ Ed. New York: The Guilford Press, 2006. 770 p.

BARROS, D.B. Os usos e sentidos do metilfenidato: experiências entre o tratamento e o aprimoramento da atenção. 2014. 182 p. Tese (Doutorado em Saúde Coletiva) - Instituto de Medicina Social, Universidade do Estado do Rio de Janeiro, Rio de Janeiro, 2014. 
BIANCHI, E. Gubernamentalidad, tecnologías y disputas. Una genealogía de los saberes profesionales acerca del ADHD en la infancia (1994-2012). 2014. 312 p. Tese (Doctorado en Ciencias Sociales) - Facultad de Ciencias Sociales, Universidad de Buenos Aires, Buenos Aires, 2014.

BIANCHI, E. et al. Medicalización más allá de los médicos: marketing farmacéutico en torno al trastorno por déficit de atención e hiperactividad en Argentina y Brasil (19982014). São Paulo, Revista Saude e Sociedade, v. 25, n. 2, p. 452-462, 2016.

BIGNONE, I.M.I.; SERRATE, M.; DIEZ, R.A. Farmacovigilancia de las drogas usadas en niños con diagnóstico de ADD/H o ADHD. In: Benasayag. L. ADDH. Niños con déficit de atención e hiperactividad. ¿Una patología de mercado? Una mirada alternativa con enfoque multidisciplinario. Argentina: Noveduc, 2007. p. 173-208.

BRASIL. Ministério da Saúde. Portaria GM no 3.088 [2011 Dez 23]. Institui a Rede de Atenção Psicossocial para pessoas com sofrimento ou transtorno mental e com necessidades decorrentes do uso de crack, álcool e outras drogas, no âmbito do Sistema Único de Saúde. Disponible en: <http://bvsms.saude.gov.br/bvs/saudelegis/gm/2011/ prt3088_23_12_2011_rep.html>.

. Ministério da Saúde. Resolução no 466, de 12 de dezembro de 2012. Diretrizes e normas regulamentadoras de Pesquisa envolvendo seres humanos. Comissão Nacional de Ética em Pesquisa (CONEP) do Conselho Nacional de Saúde. Ministério da Saúde. Disponible en: <http://bvsms.saude.gov.br/bvs/saudelegis/cns/2013/res0466_12_12_2012.html>

. Ministério da Saúde. Secretaria de Atenção à Saúde. DAPE. Coordenação Geral de Saúde Mental. Reforma psiquiátrica e política de saúde mental no Brasil. Documento apresentado à Conferência Regional de Reforma dos Serviços de Saúde Mental: 15 anos depois de Caracas. Brasília: OPAS, 2005.

CARBAJAL, M. ADD y ADHD, una mirada desde el periodismo. In: BENASAYAG, L. $A D D H$. Niños con déficit de atención e hiperactividad. ¿Una patología de mercado? Una mirada alternativa con enfoque multidisciplinario. Argentina: Noveduc, 2007. p. 237-244.

CHAGAS, B. da S. Novo transtorno, velho problema: a identificação do TDAH pelo olhar do professor. 2013. 119 p. Dissertação (Mestrado em Saúde Coletiva) - Instituto de Medicina Social, Universidade do Estado do Rio de Janeiro, Rio de Janeiro, 2013.

CONRAD, P.; BERGEY, M.R. The impending globalization of ADHD: Notes on the expansion and growth of a medicalized disorder. Social Science and Medicine, v. 122, p. 31-43, 2014.

CSILLAG C. Psychiatric reform law comes into effect in Brazil. The Lancet, v. 357, n. 9265, p. 1346, 2001.

FARAONE S. Reformas en salud mental. Dilemas en torno a las nociones, conceptos y tipificaciones. Salud Mental y Comunidad-UNLa, 2013; v. 3, n. 3, p. 29-40. 
FARAONE, S., BIANCHI, E. ADHD's journey in Argentina: from methylphenidate's rise to tensions among health professionals. In: BERGEY, M., FILLIPE, A., CONRAD, P., SINGH, I. (Eds.). Global Perspectives on ADHD: Social Dimensions of Diagnosis and Treatment in 16 Countries. London: Johns Hopkins University Press, 2016.

FÓRUM SOBREA MEDICALIZAÇÃO DA EDUCAÇÃO E SOCIEDADE. Medicalização da Vida. Jornal do Conselho Regional de Psicologia do Rio de Janeiro, n. 35, p. 8-9, 2013.

HEALY, D. The creation of psychopharmacology. Cambridge, Massachusetts: Harvard University Press, 2002. 469 p.

INTERNATIONAL NARCOTICS CONTROL BOARD (INCB). Informe 1995.

Disponible en: <http://www.incb.org/documents/Publications/AnnualReports/AR1995/ AR_1995_S.pdf>. Acceso: 28 dic 2015.

Informe 2006. Disponivel em: <http://www.incb.org/documents/Publications/ AnnualReports/AR2006/AR_2006_Spanish.pdf>. Acceso: 28 dic 2015.

. Psychotropic Substances. Statistics for 2011. Assessments of Annual Medical and Scientific Requirements. United Nations: New York: INCB, 2012.

. Psychotropic Substances. Statistics for 2012. Assessments of Annual Medical and Scientific Requirements. United Nations: New York: INCB: 2013.

Report of the International Narcotics Control Board for 2007. United Nations: New York: INCB, 2008.

ITABORAHY, C.; ORTEGA, F. O metifenidato no Brasil: uma década de publicaçôes. Ciência \& Saúde Coletiva, Rio de Janeiro, v. 18, n. 3, p. 803-816, 2010.

JANIN B. Niños desatentos e hiperactivos. ADD/ADHD. Reflexiones críticas acerca del Trastorno por Déficit de Atención con o sin Hiperactividad. Buenos Aires: Noveduc, 2004. JOSELEVICH E. AD/HD. Qué es, qué hacer. Recomendaciones para padres y docentes. Buenos Aires: Paidós, 2003.

LANGE, K.W. et al. The history of attention deficit hyperactivity disorder. ADHD Atten Def Hyp Disord, v. 2, n. 4, p. 241-255, 2010.

MANIOWICZ, D. Malos diagnósticos y sobremedicación. La infancia en peligro. Revista Veintitrés, 16 jun 2011. Disponible en: <http://veintitres.elargentino.com/nota-2927portada-titulo.html>. Acceso: 28 dic 2015.

MATTOS, P. et al. O ADHD é subtratado no país. Carta aos editores. Rev Bras Psiquiatr, v. 34, n. 4. p. 513-516, 2012.

MAYES, R.; BAGWELL, C.; ERKULWATER, J. ADHD and the Rise in Stimulant Use Among Children. Harvard Review of Psychiatry, v. 16, n. 3, p. 151-166, 2008.

MONCRIEFF, J., TIMIMI, S. Is ADHD a valid diagnostic in adults? No. British Medical Journal, v. 340, n. 7749, p. 547, 2010. 
MOYANO WALKER, J.M. ADHD ¿Enfermos o singulares? Una mirada diferente sobre el sindrome de hiperactividad y déficit de atención. Buenos Aires: Lumen, 2004.

ORTEGA, F., ZORZANELLI, RT., GONÇALVES, VP. Academic and Professional Tensions and Debates around ADHD in Brazil. In: BERGEY, M. et al. (Eds.). Global Perspectives on ADHD: Social Dimensions of Diagnosis and Treatment in 16 Countries. London: Johns Hopkins University Press, 2016.

ROSE N. Disorders without borders? The Expanding Scope of Psychiatric Practice. BioSocieties, n. 1, v. 1, p. 465-484, 2006.

SÃO PAULO (CIDADE). Portaria vo 986/2014, Secretaria Municipal de Saúde. Diário Oficial da Cidade de São Paulo, n. 109. Jun 12, 2014, p. 19.

SINGH, I. Beyond Polemics: Science and ethics of ADHD. Nature Reviews, v. 9, n. 12, p. 957-964, 2008.

STIGLITZ G. DDA, $A D D, A D H D$, como ustedes quieran. El mal real y la construcción social. Argentina: Grama, 2006.

TIMIMI, S. et al. A critique of the international consensus statement on ADHD. Clinical Child and Family Psychology Review, v. 7, n. 1, p. 59-63, 2004.

TIMIMI, S.; RADCLIFFE, N. The rise and rise of attention deficit hyperactivity disorder. Journal of Public Mental Health, v. 4, n. 2, p. 9-13, 2005.

UNTOIGLICH G. Medicalización y patologización de la vida: situación de las infancias en Latinoamérica. Nuances: estudos sobre Educação, Presidente Prudente-SP, n. 25, v. 1, p. 20-38, 2014.

WEDGE, M. A Disease Called Childhood: why ADHD became an American epidemic. New York: Avery Publishing Group, 2015. 272 p.

\section{Nota}

${ }^{1}$ E. Bianchi participou da formulação da ideia do artigo, do desenho da pesquisa na Argentina, da coleta e análise dos dados referentes à Argentina, da análise dos dados comparativos entre Argentina e Brasil, da redação do texto. S. Faraone participou da análise dos dados referentes a Argentina, do desenho da pesquisa na Argentina, da análise comparativa entre os dados de Argentina e Brasil, da revisão integral e redação do artigo. F. Ortega participou da análise dos dados referentes ao Brasil, do desenho da pesquisa no Brasil, da análise comparativa entre os dados de Argentina e Brasil, da revisão integral e redação do artigo. V.P. Gonçalves participou do desenho da pesquisa no Brasil, da coleta e análise de dados referentes ao Brasil, da redação da análise comparativa entre Brasil e Argentina e da finalização do artigo. R.T. Zorzanelli participou do desenho da pesquisa no Brasil, da análise de dados referentes ao Brasil, da análise comparativa dos dados de Brasil e Argentina, da revisão integral e redação do artigo. 


\section{Abstract}

Controversies about ADHD and methylphenidate in discussions on medicalization in Argentina and Brazil The paper aims to offer a systematic analysis of some results from investigations conducted in Argentina and Brazil relating on the use of methylphenidate, its prescription and sale's regulation. We also examined the discussions among researchers and professionals assisting individuals diagnosed with ADHD in both countries. The article integrates two research fields. In Argentina, newspaper articles, official and professional organizations' numeric data, and individual and group health professionals semi structured interviews conducted between 2007 and 2011 were analyzed. In Brazil scientific journals, participant observation and school teachers and health professionals were analyzed. Conclusions include that social movements in both countries have been organized and articulated, in an attempt to promote discussion on the medicalization of childhood and its deployment in society.

> Keywords: ADHD; methylphenidate; Argentina; Brazil; medicalization. 
Controvérsias sobre TDAH e metilfenidato nas discussões sobre medicalização na Argentina e no Brasil

$\mathrm{O}$ artigo pretende oferecer uma análise sistemática de alguns resultados das investigaçoes realizadas na Argentina e no Brasil sobre o uso de metilfenidato, sua prescriçãoo e regulamento de venda. Também examinamos as discussões entre pesquisadores e profissionais que atendem indivíduos com diagnóstico de TDAH em ambos os países. $\mathrm{O}$ artigo integra dois campos de pesquisa. Na Argentina, foram analisados artigos de jornal, dados numéricos das organizaçōes oficiais e profissionais e entrevistas semiestruturadas individuais e grupais de profissionais de saúde realizadas entre 2007 e 2011. No Brasil, foram analisadas as revistas científicas, a observação participante e professores e profissionais de saúde. Conclui-se que os movimentos sociais nos dois países foram organizados e articulados, na tentativa de promover a discussão sobre a medicalização da infância e sua implantação na sociedade.

Palavras-chave: TDAH; metilfenidato; Argentina; Brasil;
medicalização. 